Classification

Physics Abstracts

$61.16-68.35-61.72$

\title{
S.T.E.M. chemical submicron scale investigations by EELS in the field of materials science
}

\author{
Marie-Claude Cheynet, Soizic Blais, Cyril Barioz and Dominique Duly \\ Laboratoire de Thermodynamique et Physico-Chimie Métallurgiques (UA.CNRS.29) ENSEEG, BP. \\ 75, 38402 Saint-Martin d'Hères, France
}

(Received July 25, 1993; accepted May 06, 1994)

\begin{abstract}
Résumé . - Couplé à un détecteur $\mathrm{X}$, à un spectromètre de pertes d'énergie et un système de microdiffraction, le S.T.E.M. (V.G.-HB/501) constitue un outil d'analyse chimique à très haute résolution spatiale. Sur la base d'exemples empruntés à la science des matériaux - céramique supraconductrice $\mathrm{YBa}_{2} \mathrm{Cu}_{3} \mathrm{O}_{7-\delta}$, alliages d'aluminium $\mathrm{Al}-\mathrm{Mg}$, précipitation cellulaire $\alpha / \beta$ dans les alliages binaires $\mathrm{Mg}-\mathrm{Al}$ - nous illustrons l'intérêt et l'importance de la microscopie nanoanalytique pour mettre en évidence et interpréter différents comportements, pour argumenter différentes hypothèses, ou pour tester et améliorer différents modèles théoriques.
\end{abstract}

\begin{abstract}
The S.T.E.M. (V.G. HB/501) is an ideal tool to perform high spatial resolution microanalysis using techniques such as energy dispersive X-ray spectroscopy (EDXS), electron energy loss spectroscopy (EELS) and microdiffraction. On the basis of some experiments performed in the field of materials science -superconducting ceramic $\mathrm{YBa}_{2} \mathrm{Cu}_{3} \mathrm{O}_{7-\delta}$, aluminium alloys $\mathrm{Al}-\mathrm{Mg}$, cellular precipitation $\alpha / \beta$ in binary $\mathrm{Mg}-\mathrm{Al}$ alloys - we illustrate the capacity of the nanoanalytical microscopy to demonstrate and explain different material behaviours, or to test and improve theoretical models.
\end{abstract}

\section{Introduction.}

A dedicated Scanning Transmission Electron Microscope (STEM), equipped with an X-ray detector, an energy loss spectrometer and a microdiffraction system constitutes a powerful tool for obtaining chemical and structural information at a submicron scale. These performances are due to the illumination system (field emission gun, double condenser lenses, high excitation objective lens) which is capable of producing a probe whose diameter is smaller than $1 \mathrm{~nm}$ and which contains a current of about $1 \mathrm{nA}$ [1].

On the basis of three experiments performed in the field of materials science, we report in this paper the capacity of this nanoanalytical microscopy to demonstrate and explain different behaviours, to test and improve different theoretical models or to verify and confirm different hypothesis. The first study performed on the superconducting ceramic $\mathrm{YBa}_{2} \mathrm{Cu}_{3} \mathrm{O}_{7-\delta}$, consisted 
of the evaluation of the oxygen content in and between the twin planes of the twinning structure in order to explain the mechanisms which govern the twinning. The purpose of the second experiment was to determine the solute content in the walls and in the cores of the dislocation cells in strained $\mathrm{Al}-\mathrm{Mg}$ alloys, and thus to explain the influence of atoms in solid solution on the recovery kinetics. In the third case, to improve our understanding of the discontinuous precipitation process, experiments were carried out to establish concentration profiles in the $\alpha$ phase formed in $\mathrm{Mg}-\mathrm{Al}$ alloys.

\section{Experimental conditions and theoretical principles.}

The dedicated S.T.E.M. set up in Grenoble since 1982 is equipped with a Kevex X-ray detector, a serial EEL-80 V.G. spectrometer and a double axis tilt specimen cartridge. Both spectrometers are connected with a Tracor Northern 5400 computer on-line with Macintosh and using commercial and home-made software for data processing.

For each study reported below, we have used a probe smaller than $1 \mathrm{~nm}$ in diameter; according to the experiment - EDXS or EELS - the conditions of illumination and collection vary, they are determined by the size of the apertures selected.

The accuracy of the results are strongly dependent on the features of the samples, therefore for each experiment:

- the foils were heat-treated in-situ before examination, to avoid the contamination under the beam. The contamination induces an increase of the thickness, degrades the image and disrupts the energy loss information;

- the acquisitions have been carried out on zones as far as possible thinner than $50 \mathrm{~nm}$ to limit beam broadening in the sample and to reduce plural scattering.

The procedures used to perform spectra processing, are those classically used:

- in the case of EELS analysis, the standard energy loss procedure proposed by Egerton or Isaacson [2,3] was applied to quantify the core-losses. This method fits the background to each ionization edge and measures the core loss intensity $I_{k}(\beta, \Delta)$ integrated over an energy window of width $\Delta$ starting at the ionization edge. The concentration $N_{\mathrm{A}}$ of an element $\mathrm{A}$ can then be calculated using the following formula:

$$
N_{\mathrm{A}}=\frac{1}{\sigma_{k}^{\mathrm{A}}(\beta, \Delta)} \frac{I_{k}^{\mathrm{A}}(\beta, \Delta)}{I_{1}^{\mathrm{A}}(\beta, \Delta)}
$$

where $I_{1}(\beta, \Delta)$ and $I_{k}(\beta, \Delta)$ are the integrated intensities under the low energy-loss distribution and $K$ (or $L$ ) shell excitation respectively, $\sigma_{k}(\beta, \Delta)$ is the partial cross-section for $K$ (or $L$ ) inner shell excitation of $A$, calculated with the computer programs written by Egerton $[4,5]$, where $\beta$ is the collection semi-angle and $\Delta$ the energy width of the window defined on the spectra.

In practical EELS microanalysis, relative concentrations are mainly used; they can be determined according to the following relation:

$$
\frac{N_{\mathrm{A}}}{N_{\mathrm{B}}}=\frac{\sigma_{k}^{\mathrm{B}}(\beta, \Delta)}{\sigma_{k}^{\mathrm{A}}(\beta, \Delta)} \frac{I_{k}^{\mathrm{A}}(\beta, \Delta)}{I_{k}^{\mathrm{B}}(\beta, \Delta)}
$$

The volume plasmon loss energy $\left(\Delta E_{\mathrm{p}}\right)$, resulting from the excitation of the collective oscillations of the electron-gas was also examined; it has been shown to be useful for quantitative 
microanalysis in $\mathrm{Al}$ or $\mathrm{Mg}$ alloys [6, 7]. Since this is a collective excitation it depends on the density of the conduction electrons as demonstrated by Bohm, Pines, Nozières [8] and is expressed by the following relation:

$$
\Delta E_{\mathrm{p}}=\hbar \omega_{\mathrm{p}} \alpha(\text { free electron density })^{1 / 2} \quad \text { with } \omega_{\mathrm{p}}^{2}=\frac{N e^{2}}{\epsilon_{0} m}
$$

where $e$ and $m$ are the electronic charge and mass of the electron, $\hbar$ is Planck's constant divided by $2 \pi, \epsilon_{0}$ is the permittivity of the vacuum and $N$ the number of "free" $e^{-}$per volume unit .

In the particular case of a binary alloy $\mathrm{A}-\mathrm{B}, \Delta E_{\mathrm{p}}$ is proportionnal to $\sqrt{1-[\mathrm{A}]}$, and if $[\mathrm{A}]$ is lower than 10 at\%, the relation (3) can be developed and linearized in the following form:

$$
\Delta E_{\mathrm{p}}=[\mathrm{A}] x+y
$$

In measuring the plasmon energy loss corresponding to "known-composition alloys", a calibration curve can be established, from which the composition of "unknown alloys" can be deduced.

- in the case of EDXS analysis, the Cliff-Lorimer's [9] method has been used to deduce quantitative information from the spectra recorded. This approach relates the ratio of the mass concentrations of two elements $\mathrm{A}$ and $\mathrm{B}$ to the corresponding $\mathrm{X}$-ray intensity ratios $I_{\mathrm{A}} / I_{\mathrm{B}}$ through the following formula:

$$
\frac{C_{\mathrm{A}}}{C_{\mathrm{B}}}=k_{\mathrm{A} / \mathrm{B}} \cdot \frac{I_{\mathrm{A}}}{I_{\mathrm{B}}}
$$

where $k_{\mathrm{A} / \mathrm{B}}$ is a constant deduced from measurements performed on standards. The intensities are determined by calculating the area under the peaks at the FWHM (full width at half maximum) after subtraction of the background.

\section{Results.}

3.1 DETERMINATION OF THE OXYGEN CONTENT IN THE TWINNING STRUCTURE OF $\mathrm{YBa}_{2} \mathrm{Cu}_{3}$ $\mathrm{O}_{7-\delta}$ SUPERCONDUCTING CERAMIC. - Several authors $[10,11]$ have reported the influence of the twinning structure on the superconducting properties of the $\mathrm{YBa}_{2} \mathrm{Cu}_{3} \mathrm{O}_{7-\delta}$ ceramic. In order to continuously improve the properties of these materials, it is of great importance to understand the mechanisms which govern the twinning. From HREM investigations [12], it has been suggest ed that the twinning results from a modulation of the quadratic structure, and it can be imagined that the addition of oxygen atoms induces a deformation of the lattice and a structural change from quadratic to orthorhombic. The deviation $\delta$ from the ideal stoechiometry 7 , determined by iodometric titration [12], could then be explained by an increase of the oxygen vacancies in the twin planes. To verify such a hypothesis, we decided to measure the oxygen content in the twin planes and in the matrix from nanoanalytical EELS experiments.

3.1.1 Method and experimental precautions. - The samples consisted of very fine particles obtained by grinding which were dispersed on a copper grid covered with an holey carbon film. Only the twinned particles, homogeneously thinned over large zones and for which the twin planes are parallel to the electron beam, have been analyzed; these conditions are recommended to have a better signal/noise ratio and to reduce the plural scattering effects which are strongly dependent on the thickness.

Figure 1 shows a typical EELS spectrum. Only the core losses $\mathrm{Ba}-\mathrm{M}_{4}$ and $\mathrm{Ba}-\mathrm{M}_{5}$ (noted $\mathrm{Ba}$ $\mathbf{M}_{4,5}$ later) and $\mathrm{O}-\mathrm{K}$ are sufficiently well resolved to be quantified with accuracy. Moreover to 

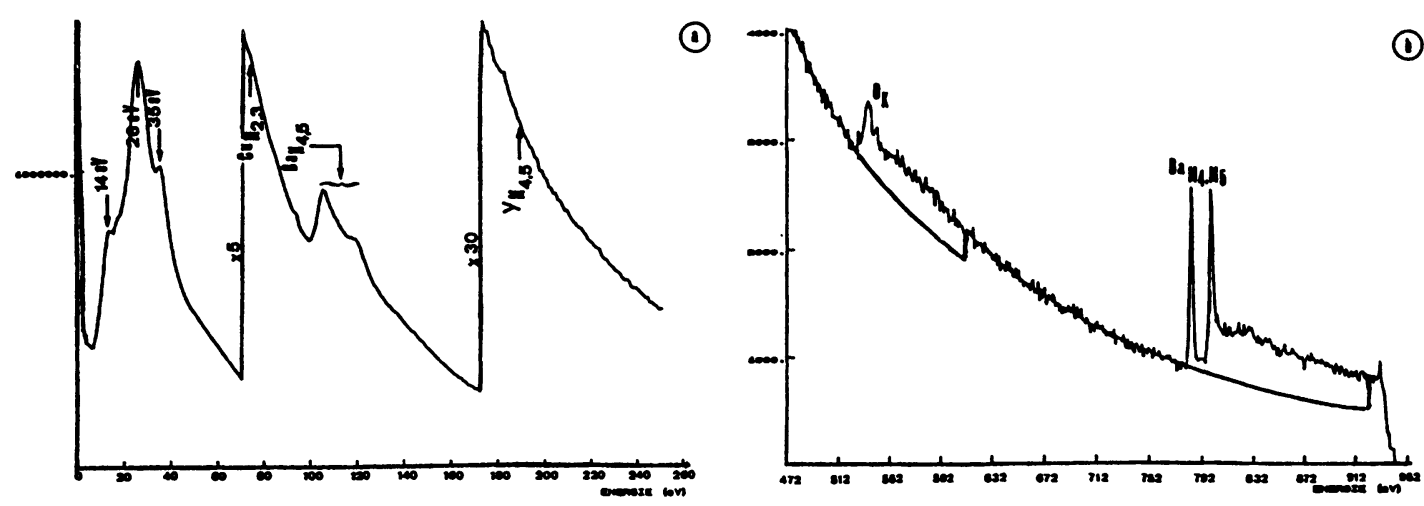

Fig. 1. - Typical electron energy loss spectrum recorded on a particle of $\mathrm{YBa}_{2} \mathrm{Cu}_{3} \mathrm{O}_{7-\delta}$ superconducting ceramic (with edges identified from the Gatan Atlas by C.C. Ahn, O.L. Krivanek). a) low energy loss domain with plasma peaks located at 14, 20 and $25 \mathrm{eV}$, and $\mathrm{Cu}-\mathrm{M}_{2,3}, \mathrm{Ba}-\mathrm{N}_{4,5}$ and $\mathrm{Y}-\mathrm{Ma}_{4,5}$ edges; b) high energy loss domain with $\mathrm{O}-\mathrm{K}$ and $\mathrm{Ba}-\mathrm{M}_{4,5}$ edges fitted by a power-law background.

limit the experimental artefacts, we have chosen to compare the ratios $I_{\mathrm{O}} / I_{\mathrm{Ba}}$ between the twin plane and the matrix, rather than the absolute intensities $I_{\mathrm{O}-\mathrm{K}}$; it is thus assumed that the $\mathrm{Ba}$ content is constant at every point in the sample, in twin planes as well as in the matrix. From preliminary EDXS experiments we have shown - as illustrated in the figure 3 - that the relative contents $\mathrm{Y}: \mathrm{Ba}: \mathrm{Cu}$ is constant in these two phases; the dispersion of the results is not statistically significant for a concentration change.

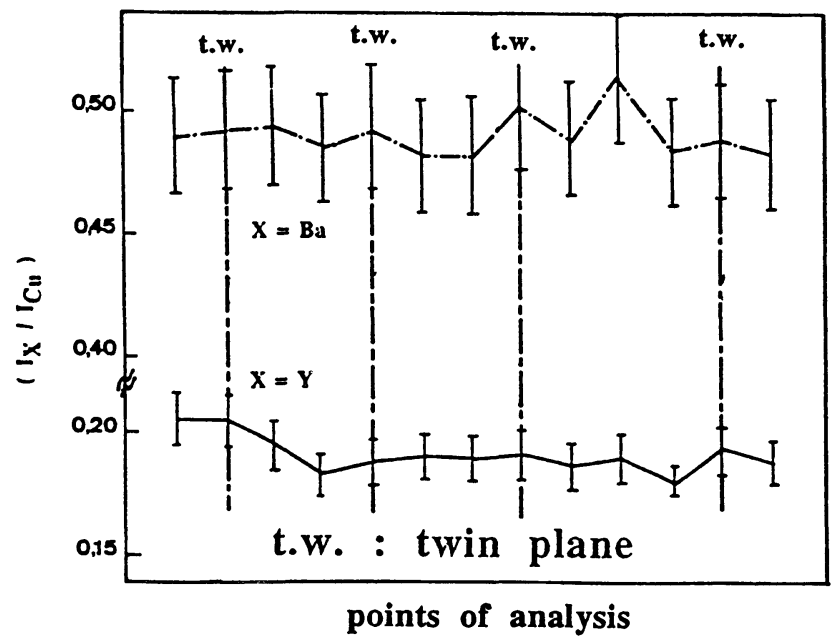

Fig. 3. - Intensities ratios $I_{\mathrm{Y}} / I_{\mathrm{Cu}}$ and $I_{\mathrm{Ba}} / I_{\mathrm{Cu}}$ as a function of the position of the probe - on or between the twin planes - recorded across a twinned particle. The variations $\mathrm{Y}: \mathrm{Ba}: \mathrm{Cu}$ are not statistically significant of a concentration change.

According to the selected twinning model and the dimension of the probe, a simple representation of the system - see figure 2 - allows to estimate of the maximum relative variation of the intensity ratio $I_{\mathrm{O}-\mathrm{K}} / I_{\mathrm{Ba}-\mathrm{M}_{4,5}}$ between the twin planes and the matrix to be approximately equal 
to $9 \%$, i.e. rather similar to the accuracy with which the intensity ratios can be determined by the electron energy loss technique. This implies that a great number of measurements has to be recorded and statistically treated to demonstrate a significant change in the oxygen content between the twin planes and the matrix.

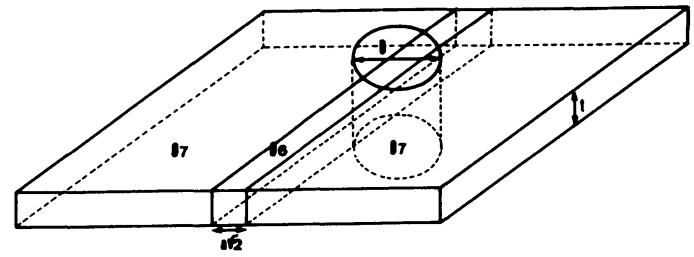

Fig. 2. - Schematic representation of the twin plane in the irradiated volume defined to calculate the relative variation of intensity ratios $-I_{\mathrm{O}} / I_{\mathrm{Ba}}$ - between twinned zones and non-twinned zones in the case of the twinning model chosen. The $\mathrm{O}_{x}$ concentration measured in the irradiated volume is related to the $\mathrm{O}_{6}$ and $\mathrm{O}_{7}$ concentrations by the expression: $x\left(\pi D^{2} t / 4\right)=6 a \sqrt{2} . D . t+7\left[\left(\pi D^{2} t / 4\right)-a \sqrt{2} . D . t\right]$, where $D$ is the diameter of the electron-probe, $t$ is the thickness of the foil and $a \sqrt{2}$ the width of the "sandwich" of the quadratic phase. The relative variation $R$ of $I_{\mathrm{O}} / I_{\mathrm{Ba}}$ between the different zones is then equal to: $R=(7-x) / x$ or $R=(4 a \sqrt{2} / 7 \pi D-4 a \sqrt{2})$, according to the beam size (10 to $15 \AA), T$ cannot exceed 0.009 .

The typical experimental conditions used to record the spectra are: incident electron beam energy: $100 \mathrm{kV}$, acquisition energy width: $1000 \mathrm{eV}$, collection semi-angle $\beta$ : 16 mrad, energy window width $(\Delta)$ for $\mathrm{O}-\mathrm{K}=76.5 \mathrm{eV}$ and for $\mathrm{Ba}-\mathrm{M}_{4,5}=145 \mathrm{eV}$, background fitting law: $A . E^{-r}$.

3.1.2 Results. - Figure 4 shows the values of the ratios $I_{\mathrm{O}-\mathrm{K}} / I_{\mathrm{Ba}-\mathrm{M}_{4,5}}$ determined in two cases: for a particle of constant thickness and for a particle of increasing thickness. It was observed that the ratios corresponding to a twin plane were always lower than those corresponding to the matrix. It was also noted that the ratio decreased when there was an increase in thickness. These results indicate clearly that the twin planes are oxygen depleted zones and confirm the hypothesis describing the twinning $\{110\}$ as a "sandwich" of a quadratic phase between two symmetric layers
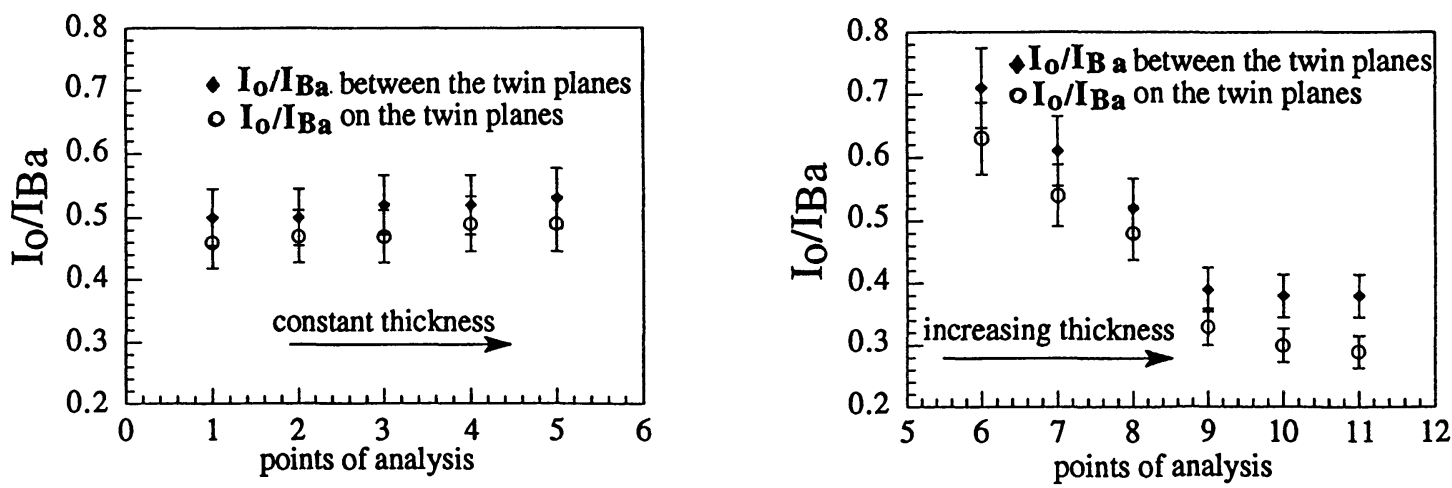

Fig. 4. - Intensity ratios $I_{\mathrm{O}} / I_{\mathrm{Ba}}$ as a function of the position of the probe - on or between the twin planes - recorded for two twinned particles: a) particle of constant thickness; b) particle of increasing thickness. 
of orthorhombic phase [13]. Further proof of this structure was obtained from the fine structure on the $\mathrm{O}_{K}$ edges; the comparison of the deconvoluted $\mathrm{O}_{\mathrm{K}}$ edges corresponding to a matrix, a twin plane and a quadratic $\mathrm{YBaCuO}_{6-\epsilon}$ showed that the $\mathrm{O}_{\mathrm{K}}$ edge corresponding to a twin plane has an intermediate shape. In the three cases the $\mathrm{O}_{\mathrm{K}}$ edges are formed with three distinct peaks located at $533 \pm 1 \mathrm{eV}, 536 \pm 1 \mathrm{eV}$ and $540 \pm 1 \mathrm{eV}$, with intensity changes according to their structure. For the orthorhombic structure the three peaks are well distinct and have the same intensity. For the quadratic structure, $\mathrm{O}_{\mathrm{K}}$ is formed of a principal peak surrounded with two small peaks. In the twin plane, an intermediate morphology is observed, which corresponds to a decrease in the oxygen content with the broadening of the mean peak. The morphology of the $\mathrm{O}_{\mathrm{K}}$ edge is directly correlated to the nature of the electronic interactions in the direction parallel to the beam ( $c$ axis in this case), therefore the broadening of the edge could be explained by an increase in the disorder of the oxygen vacancies during the structural transformation: orthorhombic to quadratic.

\subsection{MAGNESIUM SEGREGATIONS INTO DISLOCATIONS FOR Al-5 Mg ALLOYS STRAINED TO} $\epsilon=3$. - Recovery is a physical process resulting in the softening of the materials (decrease of its strength). From a microscopic point of view, recovery may be understood as an annealing-out of the dislocations introduced by cold-work and by their reorganization into low energy substructure [14]. These two mechanisms combine to reduce the internal stresses. At present the influence of atoms in solid solution on the recovery kinetics is unclear.

The experimental studies performed by C. Barioz et al. [15] on three alloys $(1,3,5 \mathrm{at} \% \mathrm{Mg})$ cold rolled $(\epsilon=3)$ and isothermally annealed at $190^{\circ} \mathrm{C}$ showed that the recovery kinetics (controlled through $\sigma_{0,2 \%}$ proof stress) occurs in two steps: a quick softening in the first minutes $\left(t_{\mathrm{c}}\right)$ followed by a slow softening over durations greater than $t_{\mathrm{c}}$. If the recovery modelisation proposed by Friedel [16] - in which dislocation annihilation is governed by cross-slip - correctly describes the second step - logarithmic decrease of stress with time - it is thus assumed that another mechanism is active in the first stage and could be due to $\mathrm{Mg}$ segregation in the dislocation walls. This is what we have tried to check by EELS experiments.

3.2.1 Method and experimental precautions. - To determine the $\mathrm{Mg}$ content in the different zones of the dislocation cells - walls and cores -, we have measured the energy of the volume plasmon loss $\left(\Delta E_{\mathrm{p}}\right)$ in order to deduce $[\mathrm{Mg}]$ as indicated in paragraph 2 . This takes advantage of:

- the high sensitivity of the volume plasmon loss energy of $\mathrm{Al}$ to small chemical additions of light elements such as $\mathrm{Mg}, \mathrm{Si}$ or $\mathrm{Li}$;

- the fact that the walls of the dislocation cells are thicker than $50 \mathrm{~nm}$, which is about 5 times greater than the spatial resolution obtained with plasmon loss microanalysis - estimated by several authors to be 10nm because of the delocalized nature of the inelastic scattering at low energy loss [17, 18]-;

- the fact that the dislocations density in the walls is higher than $10^{15} \mathrm{~cm}^{-2}$, which corresponds to an increase of the $\mathrm{Mg}$ concentration higher than $1 \%$ in the hypothesis where all the dislocations are decorated with $\mathrm{Mg}$ atoms.

In this configuration it can be thought that the presence of dislocations themselves could induce a change of the energy of the plasmon loss. Consequently we have initially estimated their real contribution using a simple calculation based on the following relation:

$$
\frac{\Delta D}{D}=3 / 2 . b^{2} \cdot \rho_{\mathrm{D}}
$$

where $b$ is the Burger's vector and $\rho_{\mathrm{D}}$ the density of dislocation. For a density of dislocations taken equal to $10^{15} \mathrm{~cm}^{-2}$ and a Burger's vector of $2.86 \AA$ in pure aluminium, an increase of the $\mathrm{Al}$ 
plasmon energy equal to $1.22 \times 10^{-4} \mathrm{eV}$ is induced, which is negligible compared to the energy change corresponding to a chemical effect.

Attention has also to be devoted to the specimen thickness which has to be chosen so that:

- dislocation cells remain stable under the beam,

- maximum signal/noise ratio is achieved (Johnson and Spence [19] claim that the specimen thickness has to be equivalent to $\lambda$ the plasmon mean free path),

- plural scattering effects are negligible.

Preliminary experiments have shown that the best compromise corresponds to a specimen thickness around $80 \mathrm{~nm}$. For greater thickness, the plural scattering effects involve a non negligible shift of the plasmon energy and for thinner samples the dislocation networks become sensitive to the beam.

Another source of plasmon energy shift may be due to surface contamination of the specimen, $\mathrm{Al}$ alloys being particularly sensitive to oxidation. Concerning the presence of an oxygen thin film on the samples and the resulting plasmon energy shift, we have considered that the contamination thickness is low and everywhere the same -in strained samples as well as in standards- and that all the spectra were recorded from areas of about equal thickness. In addition the $\mathrm{O}_{\mathrm{K}}$ signal observed on spectra recorded for each sample on an energy width equal to $1000 \mathrm{eV}$ was insignificant. Three homogeneous alloys of known compositions $(1,3,5$ at\% $\mathrm{Mg}$ ) have been previously investigated. The results obtained have allowed to establish a simple linear relation between $\Delta E_{\mathrm{p}}$ and [Mg] and also to quantify the $\mathrm{Mg}$ segregation.
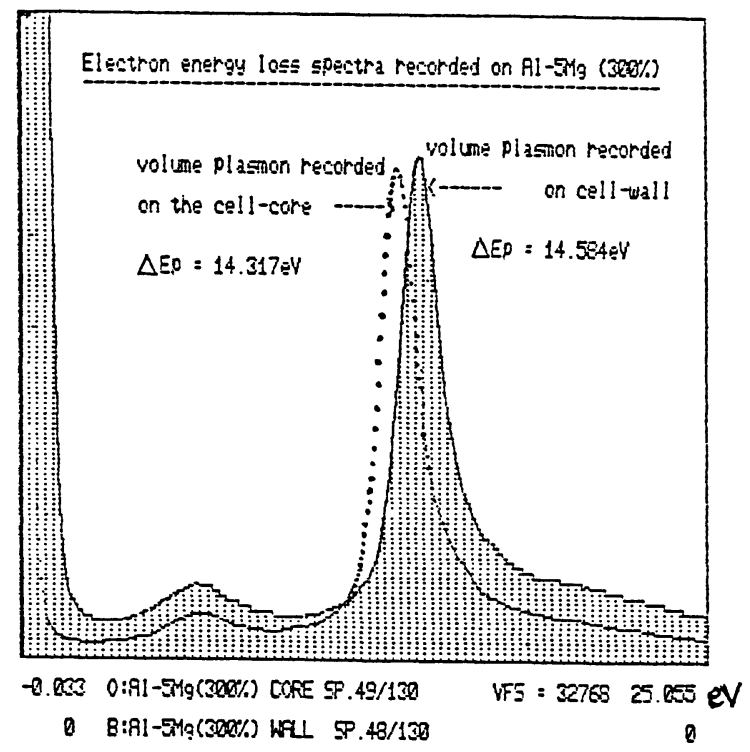

Fig. 5. - EELS spectra recorded from a strained $\mathrm{Al}-5 \mathrm{Mg}$ alloy corresponding to: a) a wall of a dislocation cell, b) a core of a dislocation cell.

The experimental conditions used for acquiring the spectra are the following: incident electron beam : $100 \mathrm{kV}$, total acquisition energy width : $20 \mathrm{eV}$ or $50 \mathrm{eV}$, collection semi-angle $\beta: 0.6 \mathrm{mrad}$, acquisition time 10 or $20 \mathrm{~s}$ per spectrum. 
3.2.2 Results. - Two typical spectra recorded on the wall and on the core of a dislocation cell are presented in figure 5. They clearly show that the energies of the volume plasmon losses corresponding to each type are different. Figure 6 shows the calibration curves obtained from the measurements performed on three homogenized Al-Mg alloys of known compositions (with 1, 3, 5 at\% $\mathrm{Mg}$ ). The equation deduced from these preliminary experiments is:

$$
\Delta E_{\mathrm{p}}=15.009-8.72[\mathrm{Mg}]
$$

Figure 7 displays the values of the volume plasmon energies recorded, in the walls and in the cores of the dislocations cells, for $\mathrm{Al}-5 \mathrm{Mg}$ alloy strained to $\epsilon=3$. The standard deviation calculated

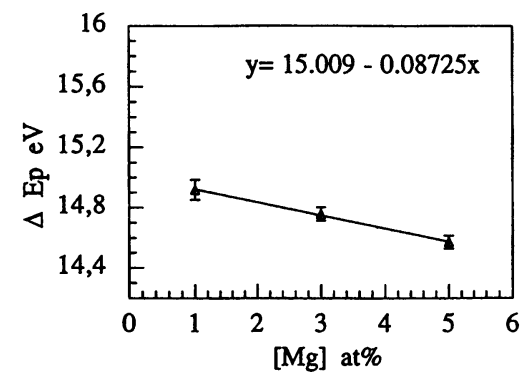

Fig. 6. - Calibration curve obtained in measuring the energy of the volume plasmon loss $\left(\Delta E_{\mathrm{p}}\right)$ as a function of the $\mathrm{Mg}$ concentration for three $\mathrm{Al}$ alloys with $1,3,5$ at\% $\mathrm{Mg}$.

from $\Delta E_{\mathrm{p}}$ measurements, is equivalent to 0.3 at $\%$ in the core cells and to 0.6 at $\%$ in the dislocation walls. These results show clearly that the walls of th e dislocation cells are enriched in $\mathrm{Mg}$, corresponding to an average content equal to 6.6 at $\%$ as compare to 5 at $\%$ in the core. They also confirm the hypothesis according to which the departure from Friedel's logarithmic law observed in the initial stage of recovery, results from the solute segregation in the dislocation walls.

\subsection{CHEMICAL MICROANALYSIS OF THE $\alpha$ PHASE FORMED IN THE CELLULAR PRECIPITATION OF} THREE Mg-Al ALLOYS. - In discontinuous precipitation, a supersaturated $\alpha^{\prime}$ phase decomposes in plates of alternating $\alpha$ and $\beta$ phases, lying parallel to the growth direction: the $\beta$ phase is homogeneous and equivalent to $\mathrm{Mg}_{17} \mathrm{Al}_{12}$, the $\alpha^{\prime}$ phase is a solute depleted phase, structurally identical to the $\alpha$ phase. The transformed zone is separated from the original by a $\mathrm{n}$ interface called the reaction front in which all involved diffusion processes take place. As the diffusion coefficient in the reaction front is finite, the $\alpha$ phase is still supersaturated at the end of the reaction.

Most theoretical and experimental work on cellular precipitation has concentrated on the steady state growth process. This process is generally modelled as the propagation of the planar reaction front at a constant velocity, perpendicularly to itself, with constant interlamellar spacing. Several attempts have been made to relate the speed of the reaction front, the interlamellar spacing $S$, and the solute profile segregation in the $\alpha$ phase. Cahn [20], has first settled the bases of the methodology and several authors - Hillert [21], Sundquist [22], B gel and Gust [23] - have proposed different models, but it has not been possible so far to discriminate between all these models.

To test the thermodynamical equations, it is necessary to determine the solute profiles between two $\beta$ lamellae and to verify that the $\alpha$ solute profile can be described by Cahn's diffusion equation. 


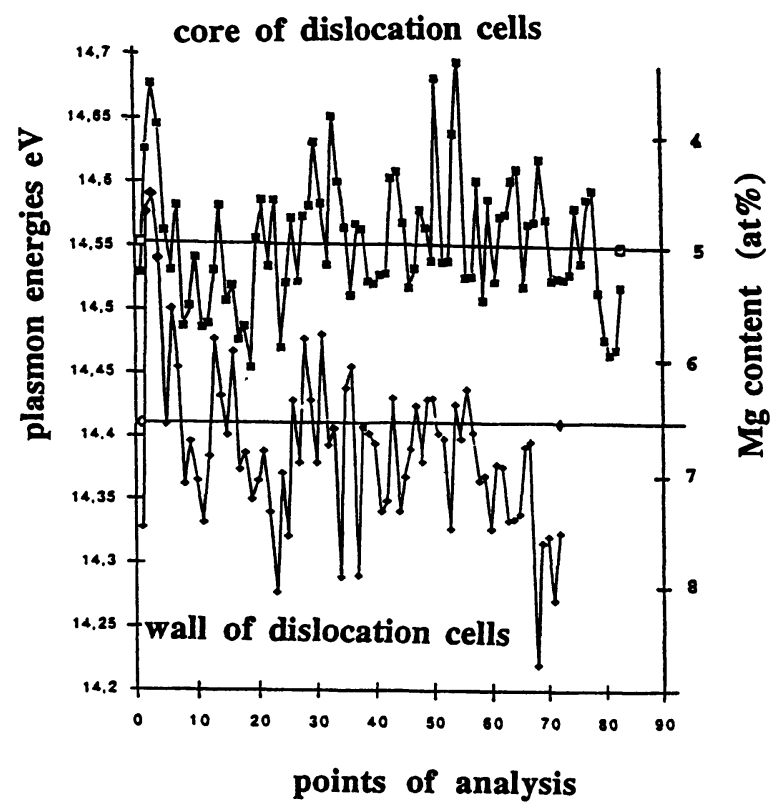

Fig. 7. - Volume plasmon energy (eV) on cell-walls (squares) and on cell-cores (lozenges) of $\mathrm{Al}-5 \% \mathrm{Mg}$, $\epsilon=3$. The average lines indicate the $\mathrm{Mg}$ concentrations measured in the walls (6.6 at\%) and in the cores (5 at\%) respectively.

With this aim, from an analytical analysis of the diffusion equation, D. Duly et al. [24] have defined a quantitative criterion according to which, if there exists a couple $\left(C_{\alpha / \beta}, \sqrt{a}\right)$ such that $\sigma(C) \leq 0.40$ at\%, the $\alpha$ solute profile obeys Cahn's diffusion equation. $C_{\alpha / \beta}$ is the solute content in $\alpha$ close to $\beta$, a is a dimensionless parameter given by Cahn [20] and Hillert [25] for a flat cell front perpendicular to the growth direction, and $\sigma(C)$ a quantitative criterion.

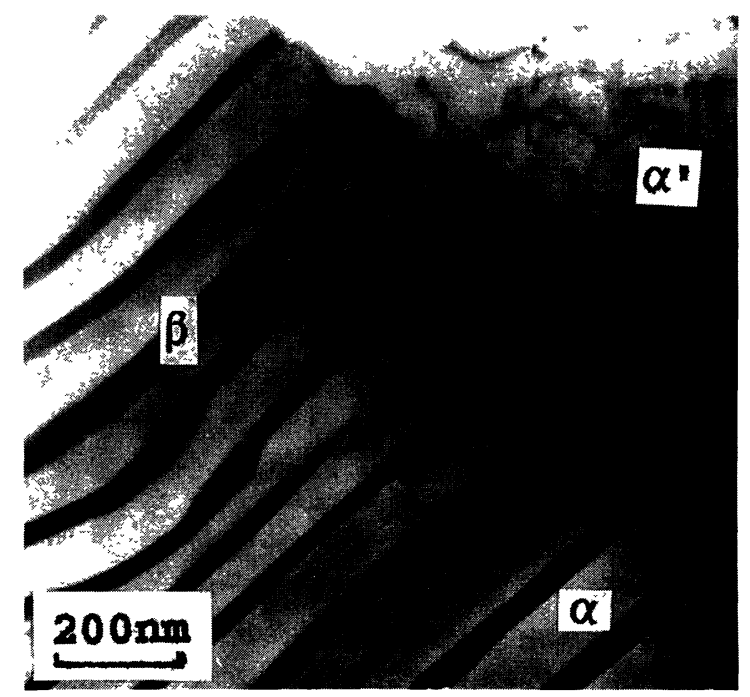

Fig. 8. - Cellular precipitation morphology. 
3.3.1 Method and experimental precautions. - The morphological aspect of the reaction product formed by discontinuous precipitation in $\mathrm{Mg}-\mathrm{Al}$ alloys is illustrated in the figure 8 .

As the $\mathrm{Mg}-\mathrm{Al}$ system exhibits dominant volume plasmon losses the analysis has been performed by relating the energy of the volume plasmon loss $\Delta E_{\mathrm{p}}$ to $\mathrm{Al}$ concentration, according to the method described in the paragraph 2.

In order to obtain a calibration of $\Delta E_{\mathrm{p}}$ in terms of composition, as previously mentioned, spectra were also recorded from three homogeneous alloys with 3, 7.7 and 10 at\% $\mathrm{Al}$; the following relation was deduced:

$$
\Delta E_{\mathrm{p}}=(10.29 \pm 0.02)+(6.8 \pm 0.4) C_{\mathrm{Al}}
$$

From these measurements a typical standard deviation equal to 0.35 at $\%$ of $\mathrm{Al}$ is calculated.

The accuracy of the results depends on the error in measuring the plasmon energy $\Delta E_{\mathrm{p}}$. Therefore to improve the results, all the profiles were recorded in zones constituted of several adjacent well-defined lamellae with low and constant thickness, and for which $\alpha / \beta$ and $\alpha / \alpha^{\prime}$ interfaces were as far as possible parallel to the electron beam. Using these conditions, the plasmon peaks are narrow and symmetric. This allowed us to measure $\Delta E_{\mathrm{p}}$ with a standard deviation equal to $0.02 \mathrm{eV}$.

For each system twenty to thirty lamellae were analysed, for each lamella two or three profiles behind the reaction front were selected, and for each profile fifteen to twenty points were recorded. Taking into account the spatial resolution obtained with plasmon loss analysis[17, 18], the distance between two consecutive points of analysis was chosen equal to $10 \mathrm{~nm}$. As with $\mathrm{Al}$ alloys, $\mathrm{Mg}$ alloys are rather sensitive to surface oxidation with the consequence of a significant shift of the plasmon peak energy over the real value. To check the possible presence of an oxide layer, spectra were systematically recorded over an energy width of $1000 \mathrm{eV}$. In these conditions the $O_{K}$ signal is insignificant, which allowed us to conclude that the plasmon energy is not shifted by the presence of an oxide surface layer.

For the profile established perpendicularly to the reaction front, the measurements are made over distances about equal to $40 / 80 \mathrm{~nm}$ on each side of the $\alpha / \alpha^{\prime}$ interface.

The experimental conditions used for recording the spectra were the following: incident electron beam energy: $100 \mathrm{kV}$, total acquisition energy width: $20 \mathrm{eV}$ or $50 \mathrm{eV}$, collection semi-angle $\beta$ : $0.6 \mathrm{mrad}$, acquisition times: 10 or $20 \mathrm{~s}$ per scan.

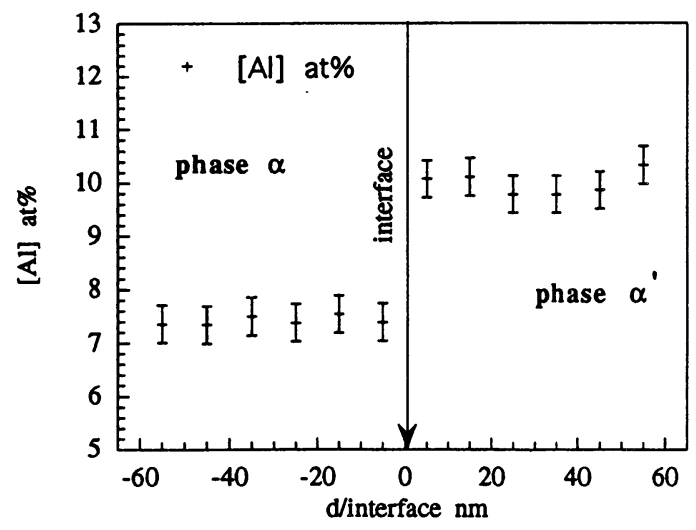

Fig. 9. - Concentration profile recorded across a cell boundary $\alpha / \alpha^{\prime}$. 
3.3.2 Results. - Figure 9 illustrates the concentration profiles obtained across the $\alpha / \alpha^{\prime}$ interfaces. It shows that the solute contents, measured $10 \mathrm{~nm}$ away from the reaction front, are equal to the nominal concentration $C_{0}$ of the studied alloy. This allows us to conclude that there is an absence of a significant volume diffusion ahead of the cell boundary, in agreement with the results previously reported by Porter et al. [26].
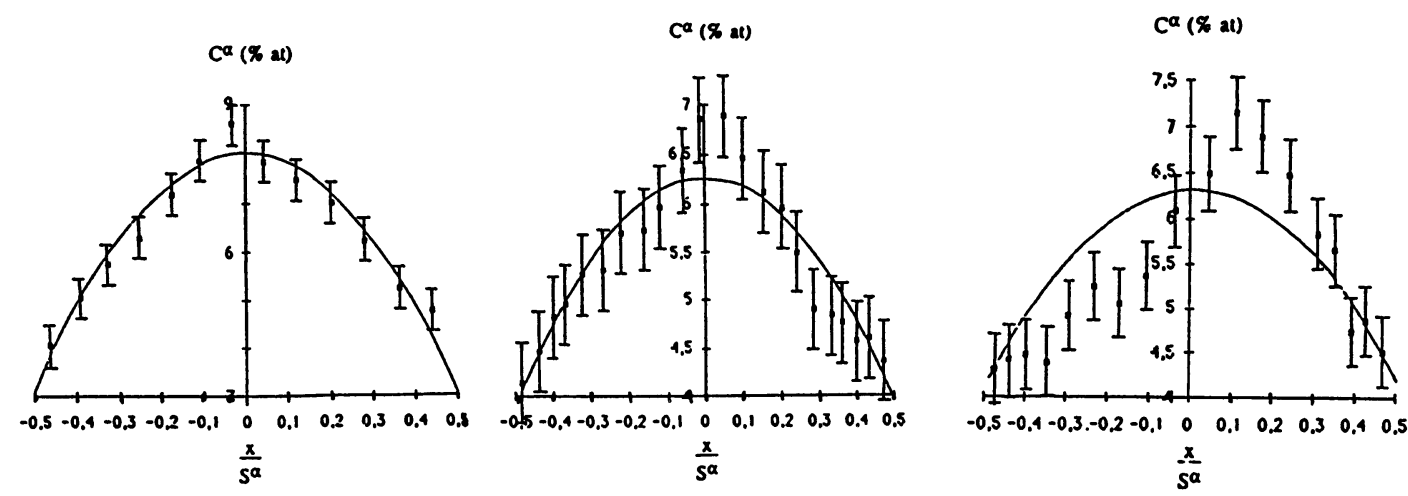

Fig. 10. - Typical concentration profiles recorded across $\alpha$ lamellae: a) symmetrical, b) hat-like, c) asymmetrical. The curve drawn for each one represents the theoretical profile calculated from Cahn's diffusion equation.

Figure 10 displays several typical concentration profiles recorded across $\alpha$ lamellae belonging to $\mathrm{Mg}$-Al alloys -with 7.7, 10 or 18.8 at\% $\mathrm{Al}$-. To normalize the results, the $\mathrm{Al}$ content is represented as a function of $x / S^{\alpha}$ where $x$ is the distance between the centre of the lamellae and the point of analysis, and $S^{\alpha}$ the width of the lamellae. These results show that the solute content decreases gradually from the centre of the lamella to the $\alpha / \beta$ interfaces when the lamellae grow parallel. They also show that asymmetrical profiles and hat-like profiles are obtained when $\alpha / \beta$ interfaces are divergent or convergent, resulting from the sudden growth arrest of a lamella, or from the nucleation of a new lamella. The curve drawn in each diagram corresponds to the theoretical profile calculated from Cahn's diffusion equation. They show that:

- symmetric experimental profiles are generally well described by a Cahn's diffusion process, contrary to asymmetric or hat- like profiles;

- Al contents at the $\alpha / \beta$ interfaces are generally greater than those corresponding to the thermodynamical equilibrium. It should be noted that the $\mathrm{Al}$ content at the interface is determined by extrapolation because it is impossible to obtain experimental data on the interface, due to the appearance of surface plasmons and overlap of $\alpha$ and $\beta$ phases caused by tilting errors. For example, for $\mathrm{Mg}-7.7$ at\% $\mathrm{Al}$ the equilibrium concentration is equal to 3.1 at\% whereas the experimental one ranges between 3.9 and 4.2 at\%; according to Aaronson and coll. [27] -for whom $C_{\alpha / \beta}$ and $C_{\mathrm{e}}$ are related by the Gibbs-Thompson's equation - or to Hillert [25] - for whom the $C_{\alpha / \beta}$ are determined by kinetics arguments - these experimental results suggest that kinetic factors are the main reasons for the discrepancy between $C_{\mathrm{e}}$ and $C_{\alpha / \beta}$.

In order to understand the relation of the asymmetry of the profiles with the non parallelism of the lamellae, a particular configuration of a cellular precipitation, observed in the $\mathrm{Mg}-10 \mathrm{at} \% \mathrm{Al}$ alloy and represented in the diagram figure 11a, has been studied in detail. In the case of the zone labelled B-I-3, two $\beta$ lamellae converge first and then diverge, elsewhere there is a $\beta$ nodule 

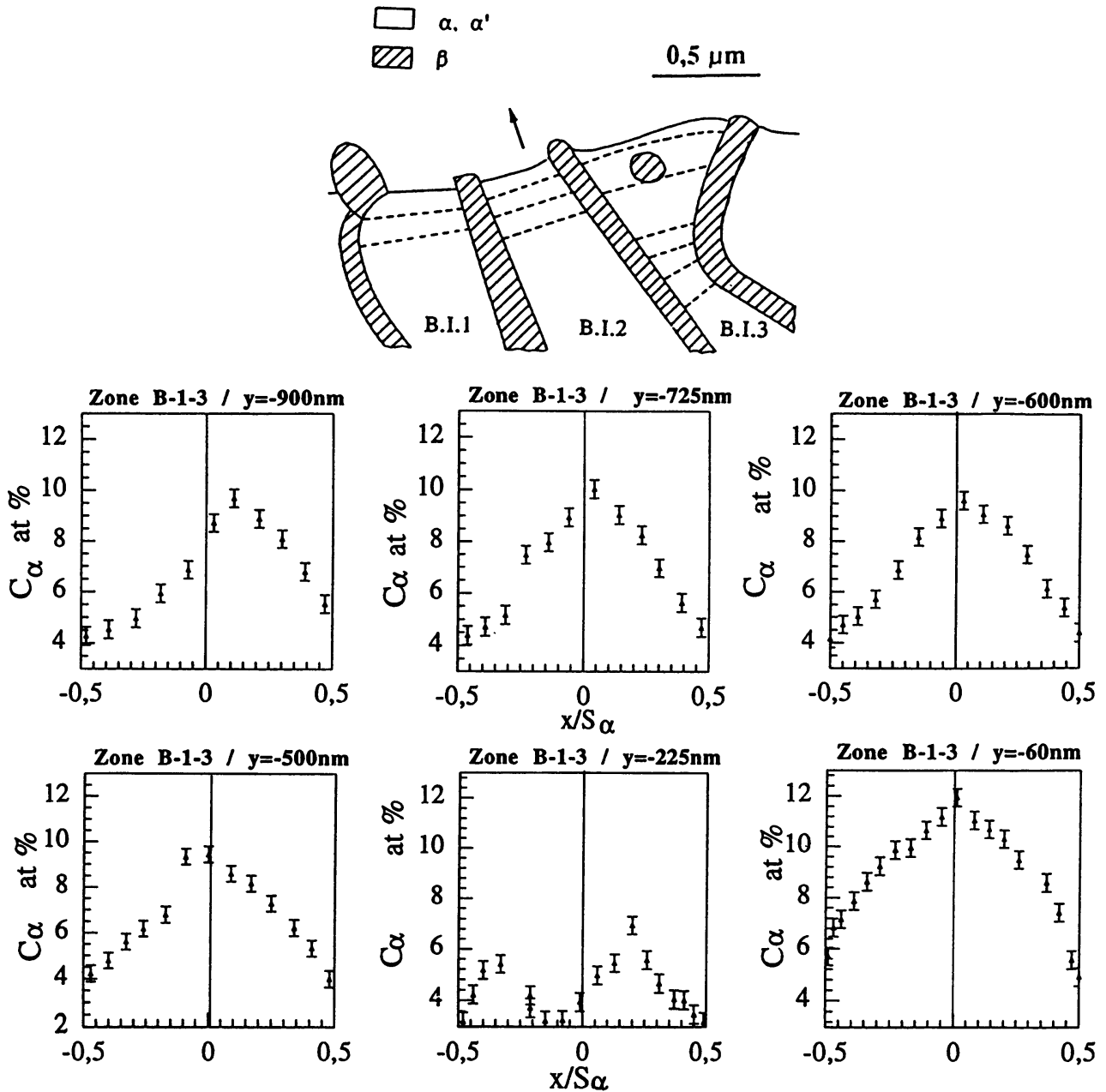

Fig. 11. - a) schematic representation of a cellular zone (noted B) constituted of pertubed $\alpha$ lamellae; the broken lines show the lines along which the profiles have been recorded. $b$ ) evolution of the concentration profiles in the $\alpha$ lamella noted B-I-3.

inside the $\alpha$ lamella. Several concentration profiles have been recorded across this lamella, they are indicated with dashed lines on the diagram. The results corresponding to each of them are presented in the figure $11 \mathrm{~b}$. It can be observed that the profiles are strongly asymmetric just before the $\beta$ lamellae diverge, and that the discrepancy with the theoretical profiles decreases to become symmetric again, in spite of the divergence.This allows to relate the asymmetry to a change of growth plane of the $\beta$ lamellae and suggests that the speed of lamellae migration is not uniform. On the other hand, the features of the profiles recorded in the surroundings of the nodule, explain clearly its formation. They show that it is the result of a very high residual sursaturation in the centre of the lamella, involving the nucleation of the $\beta$ phase.

\section{Conclusions.}

On the basis of three studies performed in the field of materials science, we have illustrated the great capacity of the FEG/STEM equipped with an electron energy loss spectrometer and an X- 
ray detector, to provide analytical data at very high spatial resolution.

The first study performed on the superconducting ceramic $\mathrm{YBa}_{2} \mathrm{Cu}_{3} \mathrm{O}_{7-\delta}$, allowed us to show that the twin planes are oxygen depleted zones and confirm the hypothesis describing the twinning $\{110\}$ as a "sandwich" of a quadratic phase between two symmetric parts of orthorhombic phase.

In the second case, from measurements of the energies of plasmon losses in the walls and in the cores of the dislocation cells formed in a strained $\mathrm{Al}$ alloy, we have determined the influence of solute atoms on the recovery kinetics, and explained the departure from Friedel's logarithmic law observed in the initial stage of the recovery.

In the last one, from numerous accurate concentration profiles determined by electron energy loss spectrometry, we have furthered in the understanding of the cellular precipitation processes, and clearly showed that Cahn's diffusion process could be invoked only when symmetrical experimental profiles are obtained.

\section{Acknowledgements.}

The authors greatly acknowledge J.C. Rouveyre for his assistance in the preparation of foils. They also want to thank Pr. P. Guyot, Pr. Y. Brechet and Dr. M. Audier for enlightening discussions, and P. Duplock for careful reading of the manuscript.

\section{References}

[1] Mory C., Ph.D Thesis, Orsay (1985).

[2] Egerton R.F., Ultramicroscopy 6 (1981) 207.

[3] Isaacson M., Johnson D., Ultramicroscopy 1 (1975) 33.

[4] Egerton R.F., Ultramicroscopy 4 (1979) 169.

[5] Egerton R.F., Scanning Electron Microsc. 1 (Chicago, 1984) 505.

[6] Williams D.B., Edington J.W., J. Microsc. 108 (1976) 113.

[7] Cundy S.L., Grundy P.J., Philos. Mag. 14 (1966) 1233.

[8] Bohm D., Pines D., Nozières D., Phys. Rev. 9 (1958) 470.

[9] Cliff G., Lorimer R.H., J. Microsc. 103 (1975) 203.

[10] Gammel C., Bishop D.J., Dolan G.J., Phys. Rev. Lett. 59 (1987) 2592.

[11] Vinnikov L.A., Gurevich L.A., Gyemelchenko G.A., Sol. State Com. (1988) 421.

[12] Blais S., Audier M., Weiss F., Sénateur J.F., ICMC'90, Garmisch Partenkirchen.

[13] Blais S., Doctorat INPG (Grenoble, 1990).

[14] Hatch J.E., Alum. Properties Phys. Metall. 1 (ASM-USA, 1984) 116.

[15] Barioz C., Bréchet Y., Cheynet M., Legresy J., Courbon J., Raynaud G., Guyot P., Third International Conference on Aluminium alloys, 2, 347, Trondheim-Norway (1992). NTH L. Arnberg, O. Lohne, E. Nes, N. Ryum Eds.

[16] Friedel J., Dislocations 1 (Pergamon Press, U.K., 1964) 273.

[17] Cundy S.L., Metherell A.J., Whelan M.J., Nicholson R.B., Proc. Roy. Soc. A307 (1968b) 267.

[18] Pennycook S.J., Ultramicroscopy 7 (1981a) 99.

[19] Johnson D.W., Spence J.C.H., J. Phys. D (Applied Phys.) 7 (1974) 771.

[20] Cahn J.W., Acta Met. 7 (1959) 18.

[21] Hillert M., Mechanism of phase transformations in crystalline solids, Institute of Metals 1 (1968) 231.

[22] Sundquist B.E., Met. Trans. 4 (1973) 1919.

[23] Bögel A., Gust W., Z. Metallkunde 79 (1988) 296.

[24] Duly D., Cheynet M.C., Bréchet Y., accepted in Acta Met. (1994).

[25] Hillert M., Met. Trans. 3 (1972) 2729.

[26] Porter D., Edington J.W., Proc. Roy. Soc. Lond. A358 (1977) 335.

[27] Aaronson H.I., Liu Y.C., Scripta Met. 2 (1968) 1. 